\title{
ON THE STRUCTURE OF SEMIDERIVATIONS IN PRIME RINGS
}

\author{
CHEN-LIAN CHUANG
}

(Communicated by Maurice Auslander)

\begin{abstract}
Let $R$ be a prime ring. By a semiderivation associated with a function $g: R \rightarrow R$, we mean an additive mapping $f: R \rightarrow R$ such that, for all $x, y \in R, f(x y)=f(x) g(y)+x f(y)=f(x) y+g(x) f(y)$ and $f(g(x))=$ $g(f(x))$. It is known that $g$ must necessarily be a ring endomorphism. Here it is shown that $f$ must be an ordinary derivation or of the form $f(x)=$ $\lambda(x-g(x))$ for all $x \in R$, where $\lambda$ is an element of the extended centroid of $R$.
\end{abstract}

As a generalization of derivations, the following notion of semiderivations was introduced in Bergen [2]:

Definition. Let $R$ be an associative ring. An additive mapping $f: R \rightarrow R$ is called a semiderivation associated with a function $g: R \rightarrow R$ if, for all $x$, $y \in R$,

(i) $f(x y)=f(x) g(y)+x f(y)=f(x) y+g(x) f(y)$;

(ii) $f(g(x))=g(f(x))$.

Assume that $R$ is a prime ring. If the semiderivation $f$ does not vanish identically on $R$, it is shown in Chang [3] that the function $g$ must necessarily be a ring endomorphism. Conversely, if $g$ is a ring endomorphism of the prime ring $R$, then the mapping $f(x)=x-g(x)$ is a semiderivation of $R$ associated with the ring endomorphism $g$. If the ring endomorphism $g$ is the identity mapping, then all semiderivations associated with $g$ are merely ordinary derivations. Our aim here is to show that any semiderivation of a prime ring $R$ assumes essentially one of these two forms:

Theorem. Let $f$ be a semiderivation of a prime ring $R$ associated with the (endomorphism) mapping $g: R \rightarrow R$. Then either one of the following two cases holds:

(1) There exists an element $\lambda$ in the extended centroid of $R$ such that $f(x)=$ $\lambda(x-g(x))$ for all $x \in R$.

(2) The endomorphism $g$ is an identity mapping and $f$ is an ordinary derivation.

Received by the editors May 19, 1989.

1980 Mathematics Subject Classification (1985 Revision). Primary 16A72, 16A70.

Key words and phrases. Semiderivation, derivation, endomorphism, extended centroid. 
Our argument is essentially a refinement of Lemma 4 [1] and is crucially based on a clever computation of this lemma.

Proof. Set $f^{\prime}(x)=x-g(x)$ for $x \in R$. Then $f^{\prime}$ is also a semiderivation of $R$ associated with the ring endomorphism $g$. Let

$$
U=\left\{\sum_{i} u_{i} f\left(x_{i}\right) v_{i}: u_{i}, v_{i}, x_{i} \in R \text { and } \sum_{i} u_{i} f^{\prime}\left(x_{i}\right) v_{i}=0\right\} .
$$

Then $U$ is obviously a two-sided ideal of $R$. Let $u_{i}, v_{i}, x_{i} \in R$ be such that $\sum_{i} u_{i} f^{\prime}\left(x_{i}\right) v_{i}=\sum_{i} u_{i}\left(x_{i}-g\left(x_{i}\right)\right) v_{i}=0$. Applying the semiderivation $f$ to $\sum_{i} u_{i}\left(x_{i}-g\left(x_{i}\right)\right) v_{i}=0$ and using the defining identities (i), (ii) for the semiderivation $f$ to expand the resulting expression, we compute, as in Lemma 4 [1]:

$$
\begin{aligned}
0 & =f\left(\sum_{i} u_{i} f^{\prime}\left(x_{i}\right) v_{i}\right) \\
& =f\left(\sum_{i}\left(u_{i} x_{i} v_{i}-u_{i} g\left(x_{i}\right) v_{i}\right)\right) \\
& =\sum_{i}\left[u_{i} f\left(x_{i} v_{i}\right)+f\left(u_{i}\right) g\left(x_{i} v_{i}\right)-f\left(u_{i} g\left(x_{i}\right)\right) g\left(v_{i}\right)-u_{i} g\left(x_{i}\right) f\left(v_{i}\right)\right] \\
& =\sum_{i}\left[u_{i} f\left(x_{i}\right) v_{i}+u_{i} g\left(x_{i}\right) f\left(v_{i}\right)+f\left(u_{i}\right) g\left(x_{i}\right) g\left(v_{i}\right)\right. \\
& =\sum_{i} u_{i} f\left(x_{i}\right) v_{i}-\sum_{i} g\left(u_{i}\right) g\left(f\left(x_{i}\right)\right) g\left(v_{i}\right) \\
& =\sum_{i} u_{i} f\left(x_{i}\right) v_{i}-g\left(\sum_{i} u_{i} f\left(x_{i}\right) v_{i}\right) .
\end{aligned}
$$

Therefore

$$
\sum_{i} u_{i} f\left(x_{i}\right) v_{i}=g\left(\sum_{i} u_{i} f\left(x_{i}\right) v_{i}\right)
$$

whenever $\sum_{i} u_{i} f^{\prime}\left(x_{i}\right) v_{i}=0$. That is, $f^{\prime}(u)=u-g(u)=0$ for all $u \in U$. If the two-sided ideal $U$ is nonzero, then by Lemma 1 [1], $f^{\prime}=0$ on $R$ and hence $g(x)=x$ for $x \in R$. Thus $g$ is the identity endomorphism of $R$ and $f$ is merely an ordinary derivation of $R$, as desired. Now, assume that $U=0$. That is, for any $u_{i}, v_{i}, x_{i} \in R, \sum_{i} u_{i} f^{\prime}\left(x_{i}\right) v_{i}=0$ implies $\sum_{i} u_{i} f\left(x_{i}\right) v_{i}=0$. Let $W$ be the two-sided ideal

$$
\left\{\sum_{i} u_{i} f^{\prime}\left(x_{i}\right) v_{i}: u_{i}, v_{i}, x_{i} \in R\right\} .
$$

Then the mapping $\phi$ defined on $W$ according to the rule

$$
\phi: \sum_{i} u_{i} f^{\prime}\left(x_{i}\right) v_{i} \rightarrow \sum_{i} u_{i} f\left(x_{i}\right) v_{i}
$$


where $u_{i}, v_{i}, x_{i} \in R$, is well defined. But $\phi$ is obviously an $(R, R)$-bimodule map of $W$ into $R$. By the definition of the extended centroid of $R$, there exists an element $\lambda$ in the extended centroid of $R$ such that $\phi(w)=\lambda w$ for all $w \in W$. In particular, for $u, v, x \in R, u f(x) v=\phi\left(u f^{\prime}(x) v\right)=$ $\lambda\left(u f^{\prime}(x) v\right)=u\left(\lambda f^{\prime}(x)\right) v$. It follows from the primeness of $R$ that $f(x)=$ $\lambda f^{\prime}(x)=\lambda(x-g(x))$ for all $x \in R$, as asserted.

\section{REFERENCES}

1. H. E. Bell and W. S. Martindale, III, Semiderivations and commutativity in prime rings, Canad. Math. Bull. 31 (4) (1988), 500-508.

2. J. Bergen, Derivations in prime rings, Canad. Math. Bull. 26 (1983), 267-270.

3. J.-C. Chang, On semiderivations of prime rings, Chinese J. Math. 12 (1984), 255-262.

Department of Mathematics, National Taiwan University, Taipei, Taiwan 10764, REPUBLIC OF CHINA 